\title{
Surgical resection and reconstruction after resection of tumors involving the sacropelvic region
}

Peter Paul Varga*, Zsolt Szoverfi, Aron Lazary

National Center for Spinal Disorders, Budapest, Hungary

National Center for Spinal Disorders, Kiralyhago u. 1-3., Budapest, Hungary, H-1126

${ }^{*}$ Corresponding author:

Peter Paul Varga

Phone: +36-1-8877900

Fax: $+36-1-8877987$

E-mail:vpp@bhc.hu 


\begin{abstract}
Objectives

Surgical management of the tumors in the sacropelvic region is a challenging field of spine surgery because of the complex local anatomy and biomechanics. Recent development in anesthesia and intensive care has allowed us to perform extended surgeries focused on the en bloc resection of sacropelvic tumors. Various techniques for the resection and for the reconstruction were published in the last decade.
\end{abstract}

\title{
Methods
}

Sacropelvic tumor resection techniques and methods for the biomechanical and soft-tissue reconstruction are reviewed in this paper.

\section{Results}

Literature data is based on case reports and case-series. Several different techniques were developed for the lumbopelvic stabilization after sacropelvic tumor resection according to three different reconstruction principles (spinopelvic fixation, posterior pelvic ring fixation, anterior spinal column fixation); however, long term follow up data and comparative studies of the different techniques are still missing. Soft-tissue reconstruction can be performed according to an algorithm depending on the surgical approach, but relatively high complication rates are reported with all reconstruction strategies. The clinical outcome of such surgeries should ideally be evaluated in three dimensions; surgical-, oncological-, and functional outcomes. The last, and most important step of the presurgical planning procedure is a careful presentation of the surgical goals and risks to the patient, who must provide a fully informed consent before surgery can proceed.

\section{Discussion}

Sacropelvic tumors are rare conditions. In the last decade, growing evidence was published on resection and reconstruction techniques for these tumors; however, experience at most medical centers is limited by the low case-number. Formation of international expert groups and initiation of multicenter studies is strongly encouraged to produce a high level of evidence in this special field of spine surgery.

Keywords: En bloc resection; Lumbopelvic stabilization; Outcome; Sacropelvic tumor; Soft tissue reconstruction 


\section{Introduction}

The sacropelvic region has got special anatomy and biomechanics therefore the surgical management of tumors in this area is one of the most challenging field of spine surgery. In addition to the complex anatomy of the bony structures ${ }^{1}$, the proximity of the large vessels, bladder and bowel makes the surgical anatomy more complicated. From the biomechanical point of view, the sacrum-sacroiliac joint-ilium complex cannot be separately discussed because the weight from the axial skeleton is transferred to the lower limbs via this area. The intact sacropelvic region is one of the key-points of the human upright posture and walking ability. Tumors involving this region alone as well as their surgical resection can significantly influence the biomechanics of the sacropelvic complex resulting in the failure of the axial load transmission. The anatomical and biomechanical consequences always have to be carefully considered during the preoperative planning to perform an oncologically and functionally optimal surgery.

\section{The role of the sacroiliac joint}

The sacroiliac (SI) joints connect the sacrum to the pelvis. This joint is a true diarhtorial joint with synovial fluid between the two large articular surfaces. In general, articular cartilage is natural, relative barriers to bone tumor spreading ${ }^{2}$, but the $\mathrm{SI}$ joint is the most common articulation invaded by tumors in the human body ${ }^{3}$. It can be related to the unique anatomy of the SI joint. First, the massive ligamentous component of the $\mathrm{SI}$ joint is exposed to tumor spreading ${ }^{3}$. Secondly, the $\mathrm{SI}$ joint is often fused and the true bony bridges between the articular surfaces can facilitate the tumor invasion ${ }^{4}$. The $\mathrm{SI}$ joint plays a key role in the biomechanics of human bipedal function connecting the spine to the lower extremities through the pelvis. The resection of the SI joint results in the dissociation of the spine with the pelvis and consequential lumbopelvic instability. However, some papers reported that motion function can be (partially) preserved in case of complete disruption of the $\mathrm{SI}$ joint $\mathrm{t}^{5,6}$, most authors agree that the restoration of the lumbopelvic stability is necessary for the early and unrestricted ambulation after sacropelvic tumor resection.

The SI joint can be invaded by tumors from the sacrum and from the iliac bone. The localization and the extent of the tumor determine the surgical technique of the resection and the need for the lumbopelvic stabilization. Previous biomechanical studies shows, that a sacrectomy extending above the S1-2 junction can significantly affect the lumbopelvic stability, thus lumbopelvic reconstruction should be considered ${ }^{7-9}$. The weakening of the pelvic ring due to the high sacral amputation can result not only in the instability in the sacropelvic junction but also in the increased risk for postoperative sacral fracture ${ }^{9}$. Based on these results, lumbopelvic stabilization should be considered in case of the following sacral tumor surgeries: high sacral amputation above S1 foramina, total sacrectomy, extended total sacrectomy and in special cases when the eccentric sacral tumor involves the SI joint only in one side (Figure 1). A pelvic tumor can also spread to the sacrum through the SI joint or the margin of the en bloc resection of the iliac tumor can be medially to the SI joint. Here, we do not discuss the special consequences of the extended internal hemipelvectomies, only mention the Type I/S hemipelvectomy when resection of the ilium is performed with the resection of a portion of the sacrum ${ }^{10}$. In these cases, the reconstruction of the sacropelvic region can be performed with unilateral constructs depending on the extent of the sacral and pelvic resection ${ }^{11-13}$. 


\section{Surgical resection of the sacropelvic tumor}

Resections of sacropelvic tumors are extended, long surgeries, challenging for the surgeon and for the patient too. The most important step of the whole process is the careful, detailed preoperative planning based on the results of the diagnostic procedure. Analysis of the histology, localization, vascularization, bony and soft tissue environment is crucial to properly plan the surgery. During the planning, the surgical approach, the type and the margins of the bony and soft tissue resection and the reconstruction strategy (stabilization and soft-tissue reconstruction) have to be considered. The description of the surgical method, the discussion of the possible complications and consequences (neurological deficit, wound healing, mobility etc) are important parts of the preoperative patient information. Last step of the preoperative preparation process is the anesthesiologist examination. The risk for the non-surgical complications because of the long anesthesia and the excessive blood loss has to be exactly evaluated and discussed with the patient.

\section{Approach}

Surgical approach depends on the localization and size of the tumor as well as on the reconstruction strategy and on the surgeon's experience. For the total sacrectomies the combined anterior and posterior approach is widely accepted, however reports about en bloc total sacrectomies from posterior-only approach have been recently published. ${ }^{14,}{ }^{15}$ High sacrectomies and resection of eccentric lesions can be mostly performed from a single posterior approach, however some authors recommend the combined anterior-posterior approach for the resection of all malignancies above $S 4{ }^{16}$. The goal of the anterior approach is the liberalization of the ventral surface of the tumor and the preparation of the vertical rectus abdominis myocutaneous (VRAM) flap if this method was chosen for the soft-tissue resection. Retroperitoneal or transabdominal approach is used to reach the tumor. After the mobilization of the rectum, the iliac vessels and ureters can be identified, mobilized and protected. The posterior branches of the internal iliac vessels, the middle and the lateral sacral vessels should be ligated with special attention to the L5 nerves. After that, the cranial plane of the resection can be determined and (if the resection plane is in the intervertebral disc space) the necessary anterior discectomy can be performed. The tumor can be separated from the anterior visceral layer with the placement of a silicon sheet. The preparation and transabdominal transfer of the VRAM flap is the last step of the anterior approach before the wound closure. Anterior and posterior surgeries can be sequentially or two-staged. For the posterior approach, different types of skin incisions are described depending on the localization and size of the tumor as well as on the soft-tissue reconstruction strategy. The goals of the posterior approach are the tumor resection and the reconstruction.

\section{Type of resection, margins}

During the surgery planning, one of the most important questions is the type of the resection. For the benign lesions, piecemeal resection of the tumor with the preservation of the nerve roots can be considered however in this case there is a greater risk for local recurrence. Malignant sacropelvic tumors should be en bloc resected with wide margins however this oncologically optimal surgical plan cannot be always executed because of technical problems or intraoperative complications. Several studies showed that en bloc resection with wide margins significantly decreased the risk for local recurrence and increased the survival of malignant sacral lesions ${ }^{17}$, but the en bloc resection 
can significantly worsen the quality of life, because of the planned sacrifice of the involved nerve roots.

\section{Bone and soft tissue resection}

After the exploration of the tumor form the posterior approach, and the wide dissection of the surrounding soft tissues, the lateral osteotomies can be performed using the threadwire saw ${ }^{18}$ or an osteotome. Wide resection of the piriformis muscles bilaterally is advised because the high rate of the local recurrence in these muscles ${ }^{19}$. After the caudal osteotomy, the coccygeal muscles and ligaments are to cut and the tumor has to be separated from the presacral anatomic structures by blunt dissection. In case of a single stage posterior surgery, this blunt, careful exploration of the (nonvisible) ventral surface is crucial. Cranial osteotomy (laminectomy and ventral osteotomy) is combined with the resection of the dural sac and nerve roots. The resected, free bony surfaces are suggested to be covered with bone wax to reduce the blood loss. After the bony resection, the completion of the soft tissue dissection has to be performed with the careful exploration of the margins of the specimen. The removal of the resected tumor has to be done with the step-by-step cranio-caudal and lateral scrolling of the specimen. With the mobilization of the specimen, the ventral structures can be identified. The rectum, the sciatic notch and any nerve roots have to be preserved if they are not involved by the tumor. The step-by-step coagulation, ligation of the presarcal - often dilated - vascular structures is crucial for hemostasis. The liberated, mobile tumorous specimen can be en bloc removed.

\section{Further considerations}

The complex anatomy of the sacropelvic region means further consideration about the resection of the tumors in this region. The proximity of the rectum can results in the tumorous involvement of the rectal wall. In this case, the en bloc tumor resection can be only performed with the combination of a rectal resection. Transient or definitive diversion colostomy has to be made in these cases before the sacral surgery. Careful bowel preparation is always crucial, high enema is advised the evening before the surgery. On the other hand, it has to be considered, that the wound infection by intestinal bacteria can occur in three ways: intraoperatively through a direct bowel injury; postoperatively as the translocation of the bacteria through the ischemic rectal wall; postoperatively from the skin because of the proximity of the anus and the fecal incontinence. Some authors suggest the preoperative insertion of ureteral stents to aid in the intraoperative identification of the ureters which can be dislocated to "unusual" place by a large tumor ${ }^{20}$. Apart of certain eccentric tumors, during the en bloc resection of a sacropelvic tumor nerve roots have to be sacrificed. The bowel and bladder function can remain quite normal if unilateral S1-S5 nerve roots are spared ${ }^{21}$. Most sacropelvic resections are associated with the bilateral resection of roots from S2 resulting in major bowel and bladder problems, saddle anesthesia and sexual dysfunction in almost all cases. The resection of S1 roots cause lower extremity muscle weakness, however ambulation (with or without aid) can be preserved even if higher lumbar roots are also sacrificed.

\section{Reconstruction}

Reconstruction of the biomechanics 
The goal of the surgical stabilization is the restoration of the load bearing structures from the lumbar spine to the pelvis. So far, there is not any method/implant for the total or partial SI joint replacement, thus all kind of stabilization is far from the natural biomechanics. Current stabilization techniques try to ensure a stable fixation between the lumbar spine and the pelvis with metal or combined systems. In some techniques, the final goal is to reach a bony fusion, but there are only few papers assessing the fusion rate in such large defects. The majority of the scientific papers are about the symmetric lumbopelvic constructs. The modification/application of the below described techniques according to the individual differences is one of the intellectual challenges with this surgery. Especially, in extended hemipelvectomies, detailed preoperative planning considering the individual biomechanics and patient-specific implant design and manufacturing can be the proper way of the future surgical management ${ }^{22}$.

In this paper, we discuss in details the lumbopelvic stabilization techniques after sacrectomy procedures. Aside from the improvised, sporadic solutions, a few stabilization techniques with different philosophy have been published. The first spinopelvic fixation (SPF) procedures were performed with the Harrington rod technology in combination of hooks and wires in the 80s before the Galveston L-rod technique with pedicle screws-iliac rods system was developed ${ }^{23}$. Several modifications of the SPF technique have been reported as well as further procedures for the posterior pelvic ring fixation (PRF) and for the anterior spinal column fixation (ACF) were developed and combined with SPF to increase the rigidity of the construction and decrease the risk for hardware related failures. Most of the clinical papers are case or case-series reports and there are some biomechanical and/or finite element studies about only experimental/theoretical techniques too. The original Galveston technique (GT) achieved the SPF with the direct implantation of the Lrods into the iliac crests (Figure 2/A). In the lumbar part, transpedicular screws implanted into three vertebrae provide the fixation of the construct, and this principle has not been changed so far. In case of modified Galveston technique (MGT) the rods are also fixed to iliac screws in the caudal part of the system (Figure 2/B, C). Yu et al. showed the biomechanical advantage of dual iliac screw fixation (Figure 2/C) compared to single iliac screw (Figure 2/B) in case of total sacrectomy and one side $\mathrm{SI}$ joint resections ${ }^{24}$. They also proved that stability of the dual-screw construct did not depend on the orientation of the iliac screws (i.e. the two screws can be placed into the same - lower - or into two different - upper and lower - iliac columns). ${ }^{25}$ Further modification of SPF was published as four rod (FRR) or double-rod double iliac screw (DDS) reconstruction technique (Figure 2/D). ${ }^{26-29}$ The biomechanical analyses showed that the additional lumbopelvic rods could increase the rigidity of the construct. On the other hand, the more metal implants the higher risk for wound healing problems. ${ }^{30}$ Varga et al. published the closed loop technique (CLT) for SPF in $2009 .{ }^{19}$ The specialty of this technique is the use of one single U-shape rod for the stabilization providing a more harmonic stress distribution along the whole construct (Figure 2/E). To increase the rigidity, the SPF was suggested to complete with the fixation of the posterior pelvic ring (PRF) by others. The triangular frame reconstruction (TFR) means that the pulled down $L 5$ vertebral body is affixed to the bilateral ilium with sacral rod (Figure 2/F). The pelvis is also connected to the spinal rods with a second sacral rod. Murakami et al. showed in their in vitro and in silico biomechanical analyses that there was less stress concentration on the implants with TFR, but an excessive stress in the iliac bones occurred which could be associated with the loosening of the sacral rod. ${ }^{31}$ Gallia et al. from the Johns Hopkins Hospital (Baltimore, Maryland) published a challenging modification of the SPF+PRF technique. ${ }^{32}$ They used an MGT for the SPF where a transiliac bar was inserted through the iliac crests. After that, 
single iliac screws were implanted and linked with a horizontal rod. The spinal (vertical) rods were attached to the transiliac bar with L-connectors. The transiliac bar, the horizontal rod and a horizontal connector between the vertical spinal rods were connected with each other using vertical connectors. Finally, a femoral allograft was placed horizontally, between the two iliac crests bridging the defect (JHU method) (Figure 2/G). Kawahara et al. published in 2003 their novel technique (NR) for the reconstruction after total sacrectomy. ${ }^{33}$ They introduced the anterior spinal column fixation (ACF) by the insertion of two pedicle screw into the inferior endplate of L5. These screws are attached to a sacral rod connecting the two iliac bones (Figure $2 / \mathrm{H}$ ). This elegant technique is a true SPF+ACF+PRF combination with the use of minimal amount of implants. With the modification of the previously mentioned closed loop technique, the vertical L5 screws can be also attached to the Ushape single rod providing further stability. ${ }^{19}$ In 2005, Dickey et al. published the use of bilateral fibular grafts for the ACF (BFFR technique). ${ }^{20}$ The fibular grafts are placed between the L5 vertebra and the bilateral iliopectineal area and the ACF can be combined with some SPF and SPF+PRF techniques (Figure 2/I). In vitro and in silico biomechanical analyses showed further rigidity of these combined systems however, the increase of the morbidity from the extension of the surgery has to be also considered.

\section{Fusion}

Lumbopelvic bony fusion could provide the final, long-term biomechanical stability after sacropelvic resection. There are two main strategies to achieve the true bony bridge between the lumbar spine and the pelvic ring. Some of the above mentioned stabilization techniques include the implantation of bone allograft as a part of the construction. In JHH technique, a horizontal femoral allograft is placed between the two iliac crests while the BFFR method implies the implantation of two fibular grafts between the $L 5$ and the pelvic ring. In the other stabilization methods, morsalized auto/allograft as well as synthetic bone grafts can be placed linking the shortest distance between the vertebra and the ilium to promote bony fusion. Pulling down of the lumbar spine with $2-4 \mathrm{~cm}$ can shorten the distance to the pelvic bone.

\section{Soft tissue reconstruction}

Resections of sacropelvic tumors are extended surgeries resulting in large tissue defect in the sacropelvic area. The en bloc resection of the tumor is often associated with the wide resection of the paraspinal and gluteal muscles, thus the proper closure of the body defect requires plastic surgical techniques. Different types of flaps can be used for the soft tissue reconstruction after resection of neoplasms along the spine ${ }^{30}$, but there are only limited possibilities at the sacropelvic area. Miles et al. in 2000, summarizing their institutional experience in the reconstruction following total sacrectomy, developed an algorithm for the management of the large sacral defects. ${ }^{34}$ Besides the description of the possible flaps, they emphasized the importance of the team work - the surgical procedure was performed by a team of surgical oncologists, neurosurgeons and plastic surgeons in their institution. They also consider the previous radiation therapy in the preoperative planning of the reconstruction technique. The later relevant publications have not changed significantly the following principles (Figure 3). ${ }^{35}$ In case of an anterior-posterior surgery and if there is no other contraindication (e.g. severe abdominal/pelvic adhesions, colostomy) the use of a vascularized vertical rectus abdominis myocutaneus flap (VRAM) is a good option. The VRAM can be transferred to the host site in transabdominal or transpelvic ways (Figure 4/A), but special care has to 
be taken to create the tunnel for the flap to prevent the pressure of the pedicle. Miles et al. used the VRAM flap in ten total sacrectomy patients and they reported wound dehistence in 4 cases, seroma and enterocutaneus fistula in 1-1 cases, but no flap necrosis. ${ }^{34}$ Glatt et al. published good results with the use of VRAM in sacropelvic defects. ${ }^{36}$ They had three slight complications (small or very small flap loss) in the total 12 cases, however the sizes of the defects were not reported. Ruggieri et al. used the VRAM in 6 of their large sacral resections with worse results. ${ }^{37}$ They had one flap necrosis and faced with postoperative infection in two other cases.

In case of single stage posterior approach, if there was no irradiation of the donor site, gluteus myocutaneous flaps can be chosen for the soft tissue reconstruction. These flaps require intact gluteal arteries. The patency of the gluteal arteries can be checked with intraoperative Doppler ultrasound and the not resected part of the gluteal muscles (GLM) with intact circulation can be advanced for the V-Y flaps bilaterally (Figure 4/B). In their paper, Miles et al. published the use of V-Y GLM flaps in 10 cases without complications while the application of rotational GLM flaps showed worse outcome ( 3 flap necrosis in 4 cases). ${ }^{34}$ Dasenbrock et al. combined the GLM flaps with the use of human acellular dermal matrix to create the pelvic diaphragm in 34 sacral resections. ${ }^{38}$ They treated four patients with posterior total sacrectomy and lumbopelvic stabilization and faced with postoperative surgical-site infection with wound dehistence in two cases, but it was not significantly more frequent than in less extended procedures.

Free flap reconstruction may be the only option if neither VRAM nor GLM can be prepared. The free flap technique (latissimus dorsi free flap, LDF, Figure 4/C) for covering large sacropelvic defect is challenging with questionable outcome because the preparation of the few adequate recipient vessels is difficult in this area. Miles et al. reported three total sacrectomy cases treated with free flap technique without severe complication, however these defects were not too large (119-170 $\left(\mathrm{cm}^{2}\right) \cdot{ }^{34}$ Garvey et al. published a significant difference in the distribution of the use of different flaps according to the size of the defect after partial sacrectomies. ${ }^{35}$ In case of the largest sacropelvic defects, they used the VRAM more frequently than the GLM flaps, however it can be also related to the anterior-posterior approach used in total sacrectomies in their institutional practice. They published a high overall complication rate $(44 \%)$ which was not significantly associated with the type of the flap or the volume of the defect.

\section{Outcome}

Clinical outcome of the sacropelvic resection surgeries has to be evaluated in three dimensions such as surgical outcome (complications), oncological outcome (survival and local recurrence) and functional outcome (disability, pain, etc). Surgical outcome can be evaluated in reflection of the intraoperative and postoperative complications which occur with high incidence these extended surgeries. In primary spinal neoplasms, oncological outcome was reported to be associated with the tumorous involvement of the resection margins several times. ${ }^{17}$ En bloc resection of the tumor with wide margins results in the lowest risk for local recurrence and systemic spread of the disease, but to achieve it can be very challenging even impossible in certain sacropelvic tumor cases. In general, functional outcome is the most important for the patient. Development of any neurologcial deficit (motor-, sensor- and vegetative disturbance) is strongly determined by the level of the nerve root sacrifice, however ambulation ability and local pain is also associated with the stability of the lumbopelvic fixation as well as the success of the soft tissue reconstruction. For the evaluation of the 
neurological outcome, the modified Biagini scale can be used ${ }^{39,40}$ however the overall functional outcome is a more complex dimension. So far, no validated measurement tool for the evaluation of the functional outcome has been published. On the other hand, the cross-culturally adapted versions of the SOSGOQ (Spine Oncology Study Group Outcomes Questionnaire) which was originally developed for metastatic spinal lesions ${ }^{41}$, seem to be an optimal tool for the follow-up of the functional outcome after sacropelvic resections too.

$\mathrm{Li}$ et al. published a case-series of 32 sacral giant cell tumors, where they used uni- or bilateral lumbopelvic stabilization in 28 cases because of the extent of the tumor resection. ${ }^{42}$ They found a significant association between the type of the resection and the oncological, surgical and neurological outcome. The intraoperative blood loss was the lowest in case of wide en bloc resections while it was the greatest in patients underwent curettage. $34 \%$ of the patients had wound complications. The local recurrence rate was significantly higher in curettage and marginal resection procedures. There was a higher rate of lower limb dysfunction in en bloc resected tumors than in curettage.

Sar et al. published a series of primary sacral tumors, where they operated three patients with tumor in the sacropelvic region. ${ }^{43}$ They reached wide margins in two cases (one GCT and one chondrosarcoma) and wide contaminated margin in one chondrosarcoma case. This latter patient died during the follow-up because of the spread of the disease while the other two patients with free margins had no local recurrence. Lumbopelvic fixation was performed using Galveston technique completed with horizontal fibular grafts between the iliac bones. All the three patients developed permanent postoperative urinary and anal incontinence and two of them had significant motor deficit. One patient had deep wound infection.

Dickey et al. published a case-series of 9 total sacrectomies. ${ }^{20}$ They performed en bloc resection with wide margins from anterior-posterior approach applying BFFR lumbopelvic stabilization in all cases. They used VRAM flaps for the soft tissue reconstruction in 6 cases. They published one wound infection and one pseudoarthrosis and hardware failure as surgical outcome and two local recurrences as well as two distant metastases as oncological outcome. Seven of the 9 patients were able to ambulate, all patients lost bowel and bladder control, none of the patients complained about significant pain.

In the didactic paper of Fourney et al., authors published a case-series of 29 en bloc sacrectomy procedures. ${ }^{39}$ They reported the outcome of five total sacrectomy and two unilateral SI joint resection because of primary bone tumors. In the former procedures, they used anterior-posterior approach, with modified Galveston stabilization and VRAM flap in four and GLM flap in one case. Four resection margins were wide, one was marginal. Three patients developed local recurrence, one of them (a chondrosarcoma patient) died of the disease. All of their patients developed complete loss of bladder and bowel control and two patients had motor weakness, however all of them were able to ambulate. They reported that the length of hospital stay (LOS) was significantly associated with the extent of sacral resection (91 days median LOS in total sacrectomy patients). The postoperative surgical complication rate was quite high (62\%) in the total cohort, and it was not significantly related to the extent of the surgery however only one total sacrectomy patient did not have any surgical complication. 
Surgical resection of sacral giant myxopapillary ependymomas was published by Quraishi et al. in $2010 .^{44}$ The six patients underwent (extended) total sacrectomy with lumbopelvic stabilization using different techniques. The soft-tissue reconstruction technique was not reported. Authors reported a range of 1.5-6.1 liters for the blood loss, development of a pseudomeningocele, wound infection, CSF fistula and pseudarthrosis in one-one case. A rare surgical complication, transient visual loss was published in one case. The bilateral posterior ischemic optic neuropathy causing this symptom can be associated with the long surgery in prone position, with the great blood loss and with the fluid overload. It is a rare complication of long spine surgeries, but it can cause permanent visual impairment. Quraishi et al. reported good functional outcome in these extended surgeries. Only one patient had moderate urinary control problems and one patient had severe, permanent low back pain after the surgery.

Papers about the posterior-only approach for en bloc sacrectomy were published by the multidisciplinary team of the Johns Hopkins University in $2012 .{ }^{14}$ In four cases, they used the JHU lumbopelvic stabilization technique with GLM flaps. ${ }^{38}$ One of the four patients was non-ambulatory after total sacrectomy. The two total sacrectomy subjects suffered from moderate bladder and bowel problems and severe pain after the surgery. The length of the surgery, blood loss and LOS were significantly associated with the extent of the surgery. The overall perioperative complication rate was $36 \%$ in their total cohort. Surgical complications were not significantly higher in total sacrectomy patients however one of these patients had major wound infection because of intraoperative bowel injury.

The better oncological outcome often means possibly worse surgical and functional outcome. The oncologically proper, radical en bloc resections of the sacropelvic tumors are extended surgeries with high intraoperative complication rate. These procedures require nerve root resections and results in large sacropelvic defect with increased risk of postoperative wound complications, local and systemic infections. Therefore, the most important step of the preoperative planning is the detailed patient's information. Some patients decide on the less radical, intralesional surgery providing better functional result even if they are aware of the worse oncological outcome.

\section{Closing remarks}

Sacropelvic tumors are uncommon conditions and the literature is rare in comprehensive reports of case-series, thus some aspects of the outcome assessment and prediction are based in low level of evidence. Recognizing the lack of evidence in this surgical field, international study groups were formed in the last years to develop multicenter databases with retrospective and prospective data about the complex management of these diseases. ${ }^{17,45}$

\section{Disclaimer Statements}

Contributors Varga PP: conception and design, drafting of manuscript, critical revision, final approval of manuscript, overall responsibility; Szoverfi Zs: literature search, critical revision; Lazary A: literature search, drafting of manuscript, figures, critical revision.

Funding The research leading to this paper received funding from the OTKA PD 104604 and the Eurospine Task Force Research.

Conflicts of interest None. 
Ethics approval No patient data is included in this manuscript and no Institutional Review Board approval is required. 
Figure 1 Surgical resection of sacropelvic tumors requiring lumbopelvic reconstruction. High sacral amputation above S1 foramina (A), total sacrectomy (B), extended total sacrectomy (C), eccentric tumors affecting the SI joint (D). Levels of nerve roots are represented (dashed line: margins of the bony resection). (based on the classification of Fourney et al. ${ }^{39}$ )
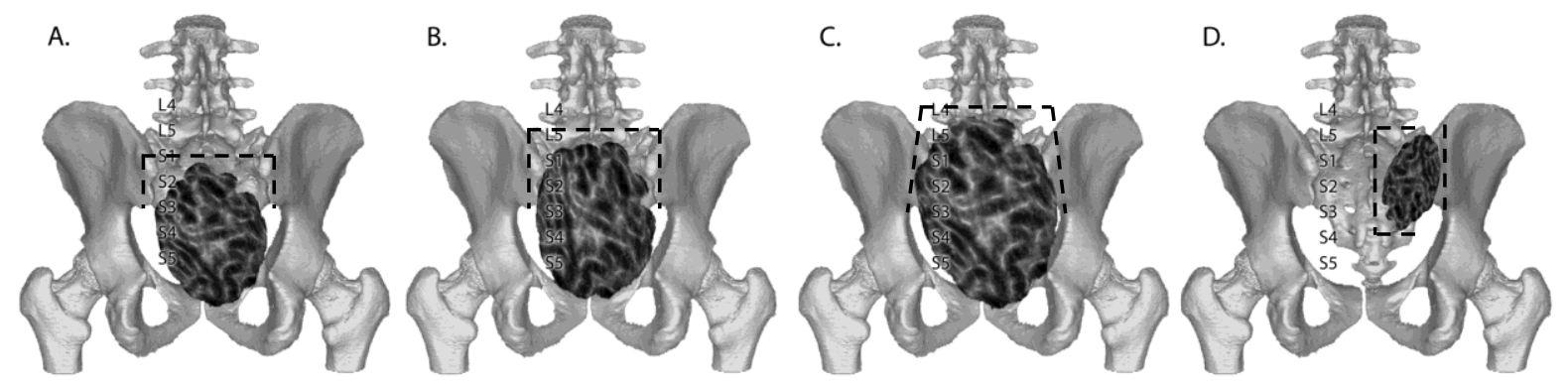

Figure 2 Lumbopelvic stabilization techniques. A: Galveston, B: modified Galveston, C: modified Galveston with two iliac screws, D: four rod, E: Closed loop, F: triangular frame, G: JHU, H: novel method, I: bilateral fibular graft
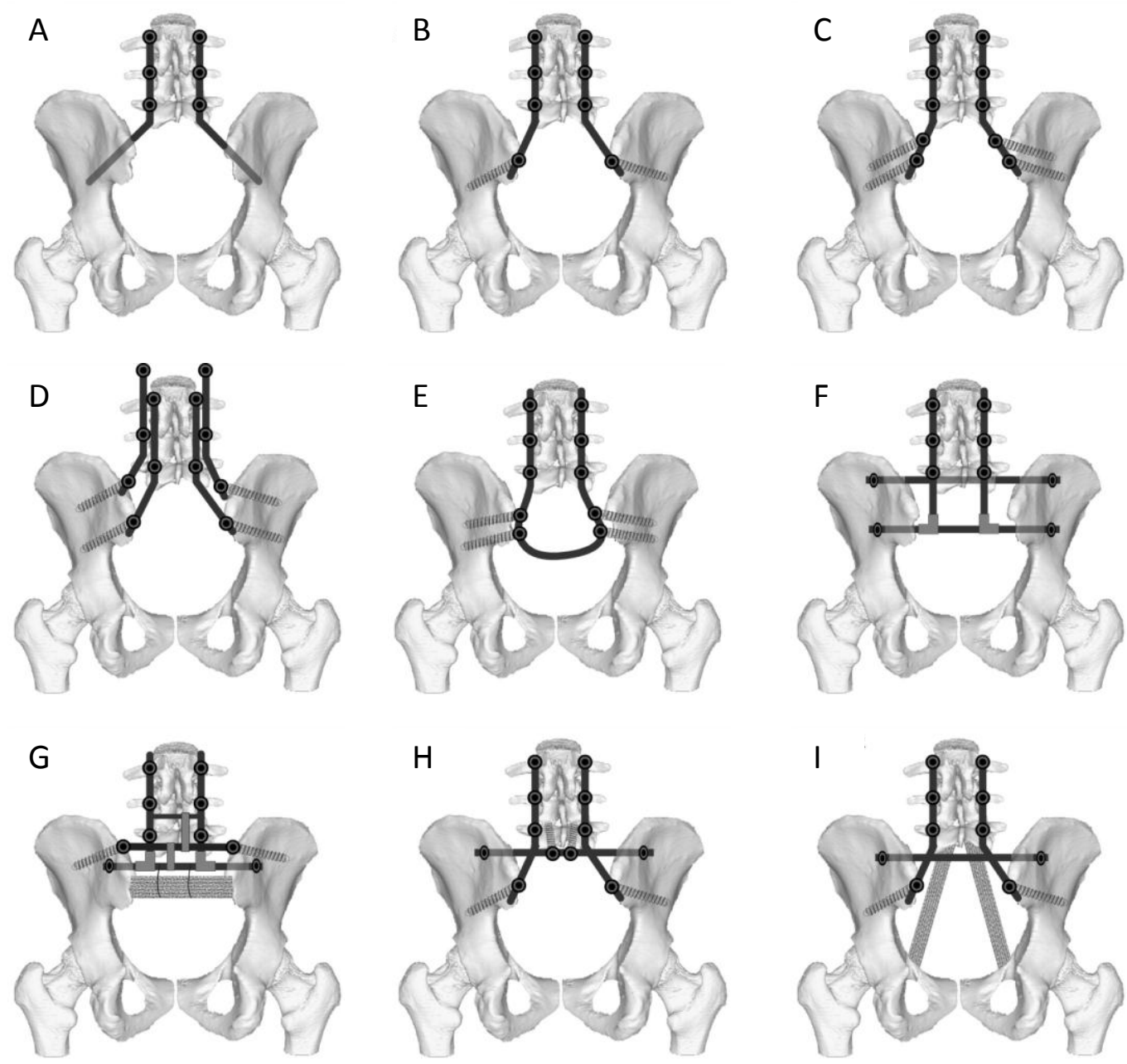
Figure 3 Soft tissue reconstruction algorithm for sacropelvic tumor resections (according to Miles et al. ${ }^{34}$ )

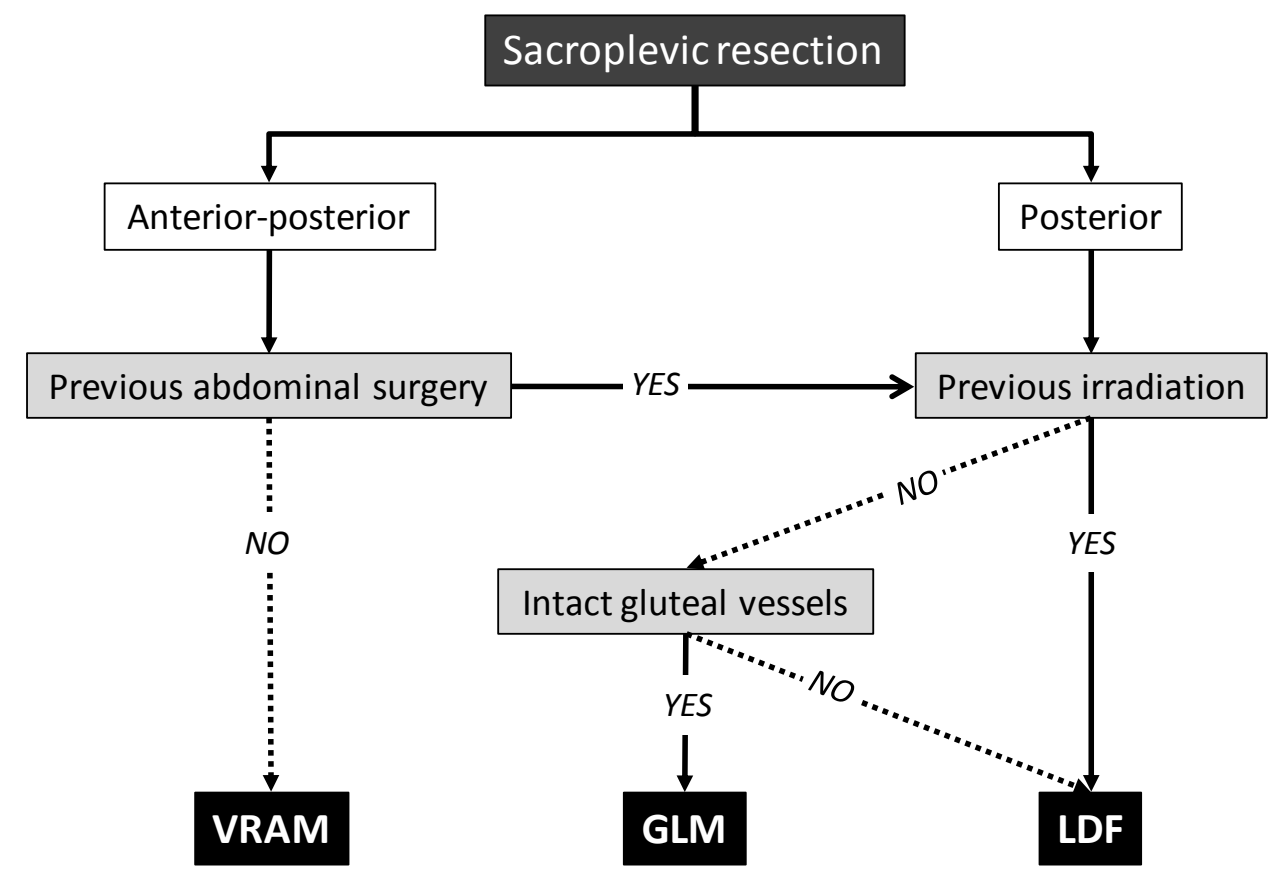

Figure 4 Reconstruction techniques for sacropelvic defects. The sacropelvic defect (black) and the flap (gray) are represented. A: vertical rectus abdominis myocutaneus (VRAM) flap (dashed line: transabdominal-, dotted line: transpelvic retroposition). B: gluteal muscle (GLM) flap, C: latissimus dorsi free (LDF) flap
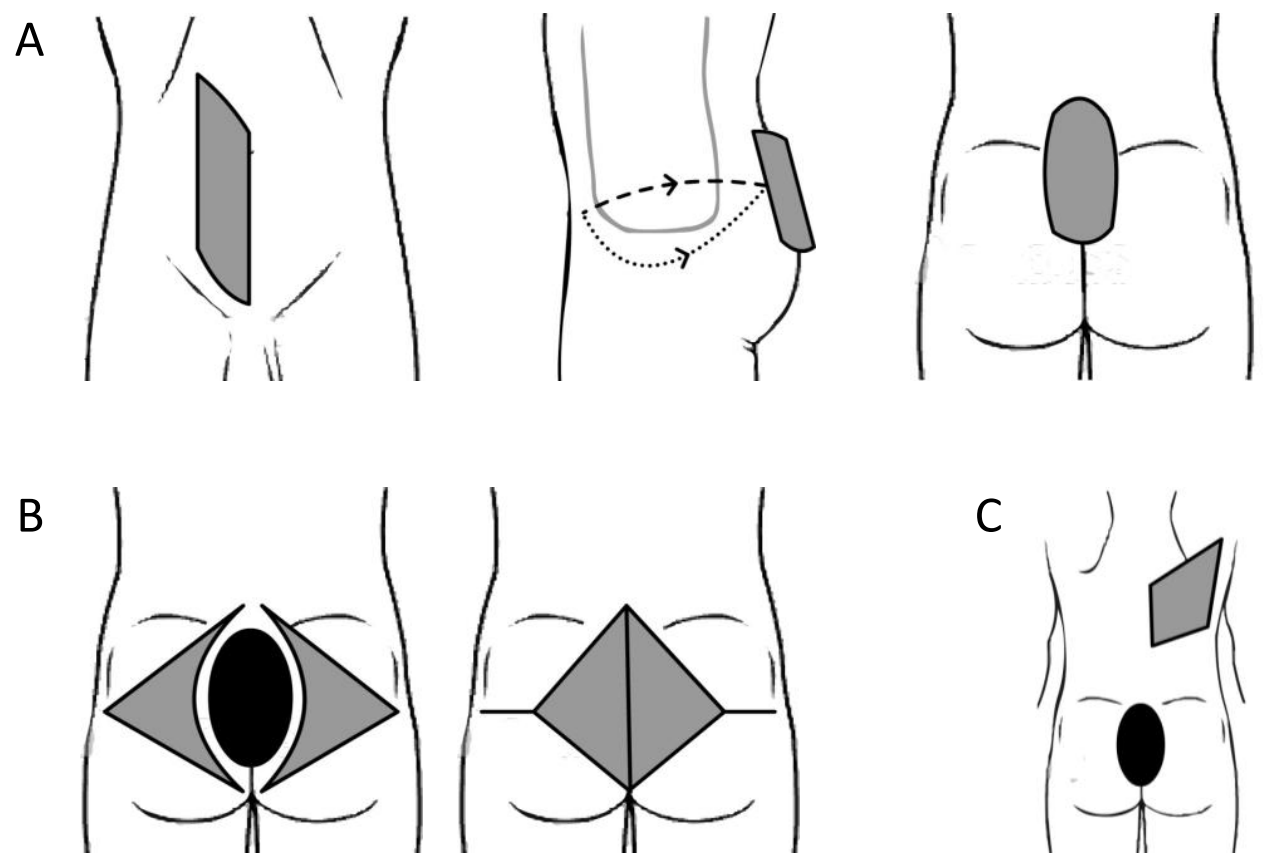


\section{References}

1. $\quad$ Cheng JS \& Song JK. Anatomy of the sacrum. Neurosurg Focus 2003; 15: E3

2. Abdelwahab IF, Miller TT, Hermann $\mathrm{G}$ et al. Transarticular invasion of joints by bone tumors: hypothesis. Skeletal Radiol 1991; 20: 279-283

3. Chhaya $\mathrm{S}$, White LM, Kandel $\mathrm{R}$ et al. Transarticular invasion of bone tumours across the sacroiliac joint. Skeletal Radiol 2005; 34: 771-777

4. Ozaki T, Rodl R, Gosheger $\mathrm{G}$ et al. Sacral infiltration in pelvic sarcomas: joint infiltration analysis II. Clin Orthop Relat Res 2003: 152-158

5. Guo Y \& Yadav R. Improving function after total sacrectomy by using a lumbar-sacral corset. Am J Phys Med Rehabil 2002; 81: 72-76

6. Wuisman $\mathrm{P}$, Lieshout $\mathrm{O}$, Sugihara $\mathrm{S}$ et al. Total sacrectomy and reconstruction: oncologic and functional outcome. Clin Orthop Relat Res 2000: 192-203

7. Gunterberg B, Romanus B, \& Stener B. Pelvic strength after major amputation of the sacrum. An exerimental study. Acta Orthop Scand 1976; 47: 635-642

8. Yu B, Zheng $Z$, Zhuang $X$ et al. Biomechanical effects of transverse partial sacrectomy on the sacroiliac joints: an in vitro human cadaveric investigation of the borderline of sacroiliac joint instability. Spine (Phila Pa 1976) 2009; 34: 1370-1375

9. Hugate RR, Jr., Dickey ID, Phimolsarnti R et al. Mechanical effects of partial sacrectomy: when is reconstruction necessary? Clin Orthop Relat Res 2006; 450: 82-88

10. Enneking WF \& Dunham WK. Resection and reconstruction for primary neoplasms involving the innominate bone. J Bone Joint Surg Am 1978; 60: 731-746

11. Court C, Bosca L, Le Cesne A et al. Surgical excision of bone sarcomas involving the sacroiliac joint. Clin Orthop Relat Res 2006; 451: 189-194

12. Wang J, Tang $\mathrm{Q}$, Xie $\mathrm{X}$ et al. Iliosacral resections of pelvic malignant tumors and reconstruction with nonvascular bilateral fibular autografts. Ann Surg Oncol 2012; 19: 40434051

13. Nassif NA, Buchowski JM, Osterman K et al. Surgical technique: Iliosacral reconstruction with minimal spinal instrumentation. Clin Orthop Relat Res 2013; 471: 947-955

14. Clarke MJ, Dasenbrock H, Bydon A et al. Posterior-only approach for en bloc sacrectomy: clinical outcomes in 36 consecutive patients. Neurosurgery 2012; 71: 357-364; discussion 364

15. Guo W, Tang X, Zang J et al. A Concise and Informative Title: One-Stage Total en bloc Sacrectomy- A Novel Technique and Report of 9 Cases. Spine (Phila Pa 1976) 2013:

16. Puri A, Agarwal MG, Shah M et al. Decision making in primary sacral tumors. Spine J 2009; 9: 396-403

17. Boriani S, Saravanja D, Yamada $Y$ et al. Challenges of local recurrence and cure in low grade malignant tumors of the spine. Spine (Phila Pa 1976) 2009; 34: S48-57

18. Tomita K \& Kawahara N. The threadwire saw: a new device for cutting bone. J Bone Joint Surg Am 1996; 78: 1915-1917

19. Varga PP, Bors I, \& Lazary A. Sacral tumors and management. Orthop Clin North Am 2009; 40: 105-123, vii

20. Dickey ID, Hugate RR, Fuchs B et al. Reconstruction after Total Sacrectomy. Clin Orthop Relat Res 2005; \&NA;: 42-50

21. Guo Y, Palmer JL, Shen L et al. Bowel and bladder continence, wound healing, and functional outcomes in patients who underwent sacrectomy. J Neurosurg Spine 2005; 3: 106-110

22. Sun W, Li J, Li Q et al. Clinical effectiveness of hemipelvic reconstruction using computeraided custom-made prostheses after resection of malignant pelvic tumors. J Arthroplasty 2011; 26: 1508-1513

23. Allen BL, Jr. \& Ferguson RL. The Galveston technique of pelvic fixation with L-rod instrumentation of the spine. Spine (Phila Pa 1976) 1984; 9: 388-394 
24. Yu BS, Zhuang XM, Li ZM et al. Biomechanical effects of the extent of sacrectomy on the stability of lumbo-iliac reconstruction using iliac screw techniques: What level of sacrectomy requires the bilateral dual iliac screw technique? Clin Biomech (Bristol, Avon) 2010; 25: 867872

25. Yu BS, Zhuang XM, Zheng ZM et al. Biomechanical advantages of dual over single iliac screws in lumbo-iliac fixation construct. Eur Spine J 2010; 19: 1121-1128

26. Shen $\mathrm{FH}$, Harper $\mathrm{M}$, Foster WC et al. A novel "four-rod technique" for lumbo-pelvic reconstruction: theory and technical considerations. Spine (Phila Pa 1976) 2006; 31: 13951401

27. Kelly BP, Shen FH, Schwab JS et al. Biomechanical testing of a novel four-rod technique for lumbo-pelvic reconstruction. Spine (Phila Pa 1976) 2008; 33: E400-406

28. Zhu R, Cheng $L M, Y u Y$ et al. Comparison of four reconstruction methods after total sacrectomy: a finite element study. Clin Biomech (Bristol, Avon) 2012; 27: 771-776

29. Mindea SA, Chinthakunta S, Moldavsky M et al. Biomechanical comparison of spinopelvic reconstruction techniques in the setting of total sacrectomy. Spine (Phila Pa 1976) 2012; 37: E1622-1627

30. Chang DW, Friel MT, \& Youssef AA. Reconstructive strategies in soft tissue reconstruction after resection of spinal neoplasms. Spine (Phila Pa 1976) 2007; 32: 1101-1106

31. Murakami $\mathrm{H}$, Kawahara $\mathrm{N}$, Tomita $\mathrm{K}$ et al. Biomechanical evaluation of reconstructed lumbosacral spine after total sacrectomy. J Orthop Sci 2002; 7: 658-664

32. Gallia GL, Haque R, Garonzik I et al. Spinal pelvic reconstruction after total sacrectomy for en bloc resection of a giant sacral chordoma. Technical note. J Neurosurg Spine 2005; 3: 501506

33. Kawahara N, Murakami H, Yoshida A et al. Reconstruction after total sacrectomy using a new instrumentation technique: a biomechanical comparison. Spine (Phila Pa 1976) 2003; 28: 1567-1572

34. Miles WK, Chang DW, Kroll SS et al. Reconstruction of large sacral defects following total sacrectomy. Plast Reconstr Surg 2000; 105: 2387-2394

35. Garvey PB, Rhines LD, Feng $L$ et al. Reconstructive strategies for partial sacrectomy defects based on surgical outcomes. Plast Reconstr Surg 2011; 127: 190-199

36. Glatt BS, Disa JJ, Mehrara BJ et al. Reconstruction of extensive partial or total sacrectomy defects with a transabdominal vertical rectus abdominis myocutaneous flap. Ann Plast Surg 2006; 56: 526-530; discussion 530-521

37. Ruggieri $P$, Angelini $A$, Pala $E$ et al. Infections in surgery of primary tumors of the sacrum. Spine (Phila Pa 1976) 2012; 37: 420-428

38. Dasenbrock HH, Clarke MJ, Bydon A et al. Reconstruction of extensive defects from posterior en bloc resection of sacral tumors with human acellular dermal matrix and gluteus maximus myocutaneous flaps. Neurosurgery 2011; 69: 1240-1247

39. Fourney DR, Rhines LD, Hentschel SJ et al. En bloc resection of primary sacral tumors: classification of surgical approaches and outcome. J Neurosurg Spine 2005; 3: 111-122

40. Biagini $\mathrm{R}$, Orsini $\mathrm{U}$, Demitri $\mathrm{S}$ et al. Osteoid osteoma and osteoblastoma of the sacrum. Orthopedics 2001; 24: 1061-1064

41. Street J, Lenehan B, Berven S et al. Introducing a new health-related quality of life outcome tool for metastatic disease of the spine: content validation using the International Classification of Functioning, Disability, and Health; on behalf of the Spine Oncology Study Group. Spine (Phila Pa 1976) 2010; 35: 1377-1386

42. Li G, Fu D, Chen K et al. Surgical strategy for the management of sacral giant cell tumors: a 32-case series. Spine J 2012; 12: 484-491

43. Sar C \& Eralp L. Surgical treatment of primary tumors of the sacrum. Arch Orthop Trauma Surg 2002; 122: 148-155

44. Quraishi NA, Wolinsky JP, Bydon A et al. Giant destructive myxopapillary ependymomas of the sacrum. J Neurosurg Spine 2010; 12: 154-159 
45. Fisher CG, DiPaola CP, Ryken TC et al. A novel classification system for spinal instability in neoplastic disease: an evidence-based approach and expert consensus from the Spine Oncology Study Group. Spine (Phila Pa 1976) 2010; 35: E1221-1229 